\title{
Integrated Information Visualization to Support Decision Making for Health Promotion in Chonburi, Thailand
}

\author{
Puangrat JINPON $^{1,2, *}$, Mullica JAROENSUTASINEE ${ }^{1}$ \\ and Krisanadej JAROENSUTASINEE ${ }^{1}$ \\ ${ }^{1}$ Center of Excellence for Ecoinformatics, School of Science, Walailak University, \\ Nakhon Si Thammarat 80160, Thailand \\ ${ }^{2}$ Information Technology and Educational Media Center, Southern College of Technology, \\ Nakhon Si Thammarat 80110, Thailand
}

("Corresponding author's e-mail: jpuangrat@gmail.com)

Received: 2 February 2016, Revised: 24 October 2017, Accepted: 4 November 2017

\begin{abstract}
A visualization of comprehensive data on health care enhances performance of decision-making in health promotion programs. We describe the design and prototype of a Dashboard Decision Support System (DDSS) as a web-based advanced tool for executives and health officers to plan and generate effective programs in health promotion and disease prevention. Data were obtained from 10 communities surrounding oil refinery, using Family and community Assessment Program (FAP), in operation since 2010. The system was developed using web-based technology and open standards, i.e., MySQL, PHP, Highcharts JS, and Google Maps. Perception of the system and its effectiveness were evaluated using a questionnaire after participants had had an approximately one month period of experience of using the system. The responses to the questionnaire were positive about the system features and system process. Using the DDSS, executives and health officers can deploy effective and appropriate programs to enhance health care in their communities.
\end{abstract}

Keywords: Integrated information visualization, data visualization, integrated information system, clinical informatics, clinical research informatics

\section{Introduction}

Over the past decade, there have been increasing disease rates and, conversely, limited funding situations [1]. This situation has driven a use of approved strategies to improve population health [1]. For instance, health promotion strategies emphasize empowering local communities to change their environments to their advantage [2]. Since political, economic, social, cultural, environmental, and biological factors can all favor or be harmful to health, 3 aspects of community based health (lifestyle, environment, and organized health services) have been focused on [3]. Lifestyles are changed as a result of emphasizing health promotion in the community, and by changing socioeconomic-political structures in the local surroundings $[3,4]$.

Moreover, the World Health Organization (WHO) has recognized growing evidence that health promotion and preventive health approaches are effective in improving overall health and well-being, reducing the burden on health care services of chronic disease and injury, addressing health inequities, simplifying the better use of resources, and enhancing economic productivity [5]. Increased emphasis on evidence and outcomes-based practice in health care has created a need for health care institutions and systems to enhance medical decision-making, via a systematic integration of data from a variety of disparate sources, in order to improve decisions for reducing morbidity and mortality, and improving 
http://wjst.wu.ac.th

patient outcomes and quality of care [6]. Therefore, a visualization of comprehensive data on health care enhances performance of decision-making for health promotion and disease prevention programs [6].

Data visualization is an essential part of Business Intelligence (BI) concept [7,8]. In health care, it is an analytical technique which represents an array of health care data in graphical format, making it easily and quickly understood [6,8]. This technique has generated a useful tool to obtain reliable epidemiological data, social demographic measures, and environmental health measures, at a high spatial resolution [9]. These benefits are obtained due to the inclusion of mathematical and algorithmic based processing of data resources, such as data mining, natural language processing, and visual analytics, to create predictive, descriptive, and prescriptive models to enable the analysis of data from user's insight [7-9]. Although various studies have been conducted to develop health information system tools in various populations $[1,6,10-13]$, the systems are undesirable powerful tools to support decision making efforts, especially in Thailand.

The aims of this study were to develop a visual Dashboard Decision Support System (DDSS), and to explore its applicability to measure and create health maps for improving health system performance, and in planning to generate practical programs in health promotions and disease preventions for communities surrounding oil refinery.

\section{Materials and methods}

\section{Study setting}

Since October 2010, the Community Health and Learning Center (CHLC), Chonburi, Thailand [14], has been conducting a population-based survey from 10 communities surrounding oil refinery: Ban Ao Udom, Ban Thung, Ban Laem Chabang, Ao Udom Market, Ban Chak Yai Chin, Ban Nam Sap, Wat Manorom, Ban Chak Krapok, Nong Manao, and Laem Thong. The amount of 15,435 individuals participated in the program, from a total population of 18,380 individuals.

\section{The Dashboard Decision Support System (DDSS) framework for the CHLC}

Visualization was integrated into the Community Health Information System (CHIS) in the form of DDSS. The CHIS was developed using a holistic health system based on Dr. Prawase Wasi's idea [15]. He stated that, "Health means perfect happiness in physical, mental and social aspects, which is the ultimate goal of life and development, and the linkage of all factors affecting health is called a "health system" [16].

The CHIS provides a major application of a knowledge discovery system for the executives and health officers of the CHLC, for clinical decision support in planning, screening, and improving individuals and communities, for healthy family monitoring, and for predictive risk assessment. Further, teachers and nursing internships from 2 universities in Chonburi have used these data for creating health maps and making activities to train and provide knowledge to communities about their current issues.

Data were obtained from the Questionnaire of the Health Information Surveys (QHIS), adapted from the Family and Community Assessment Program (FAP). FAP was first implemented in Pakpoon sub-district, Nakhon Si Thammarat, and later implemented in all regions of Thailand [17]. Personal profile data used were house location, age, marital status, weight, height, occupation, number of family members, functionality of the family, and family history of Diabetes Mellitus (DM), hypertension, and genetic disorder. If there were elderly people in the house aged 55 or above, the Barthel ADL index assessment was conducted. A total 832 elderly people from 8 communities were interviewed and classified into 3 groups: (1) severe disability, (2) moderate disability and (3) no disability. The Barthel $\mathrm{ADL}$ indices ranged from 0 (fully dependent) to 20 (independent) [18]. 
(a)

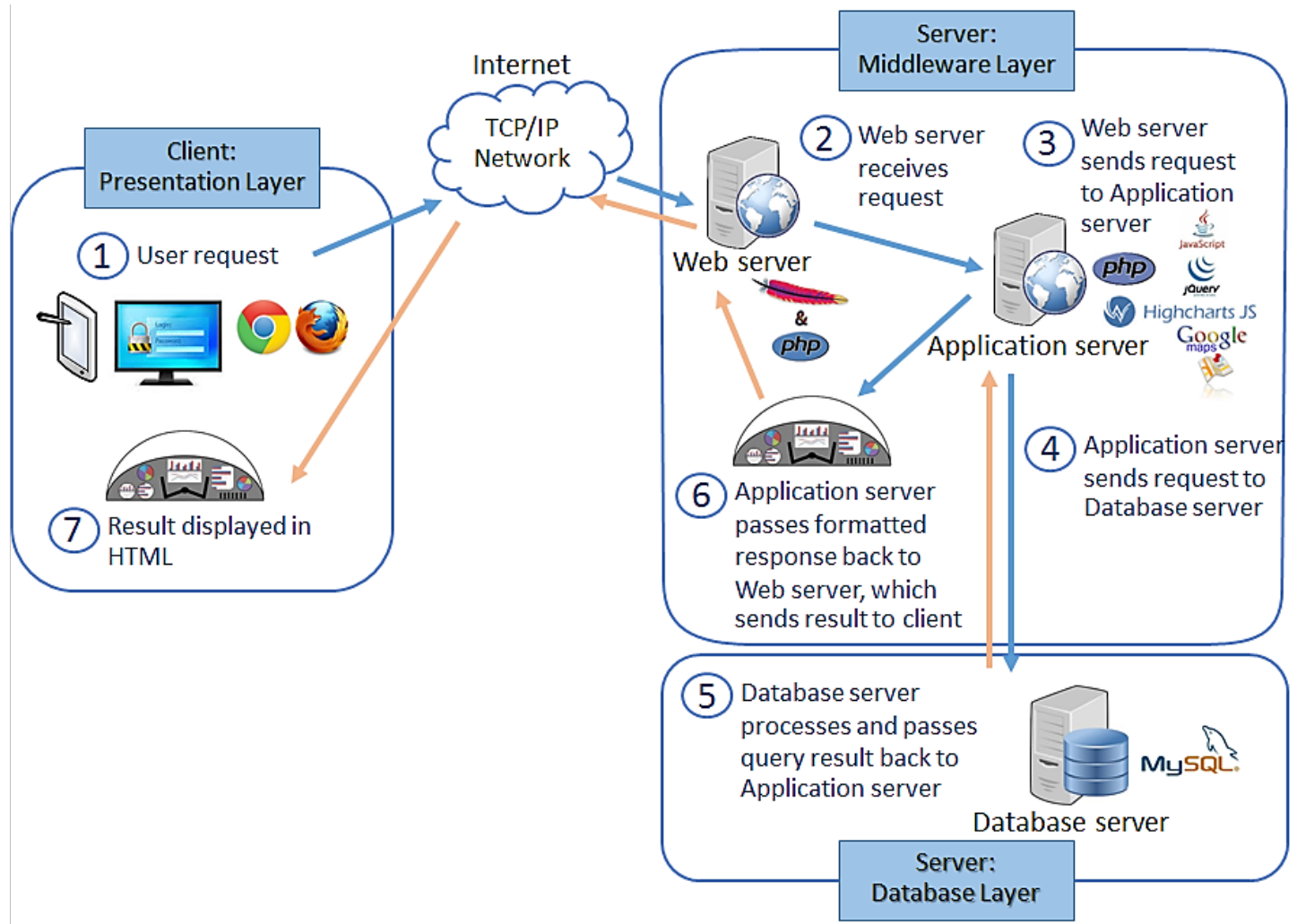

(b)

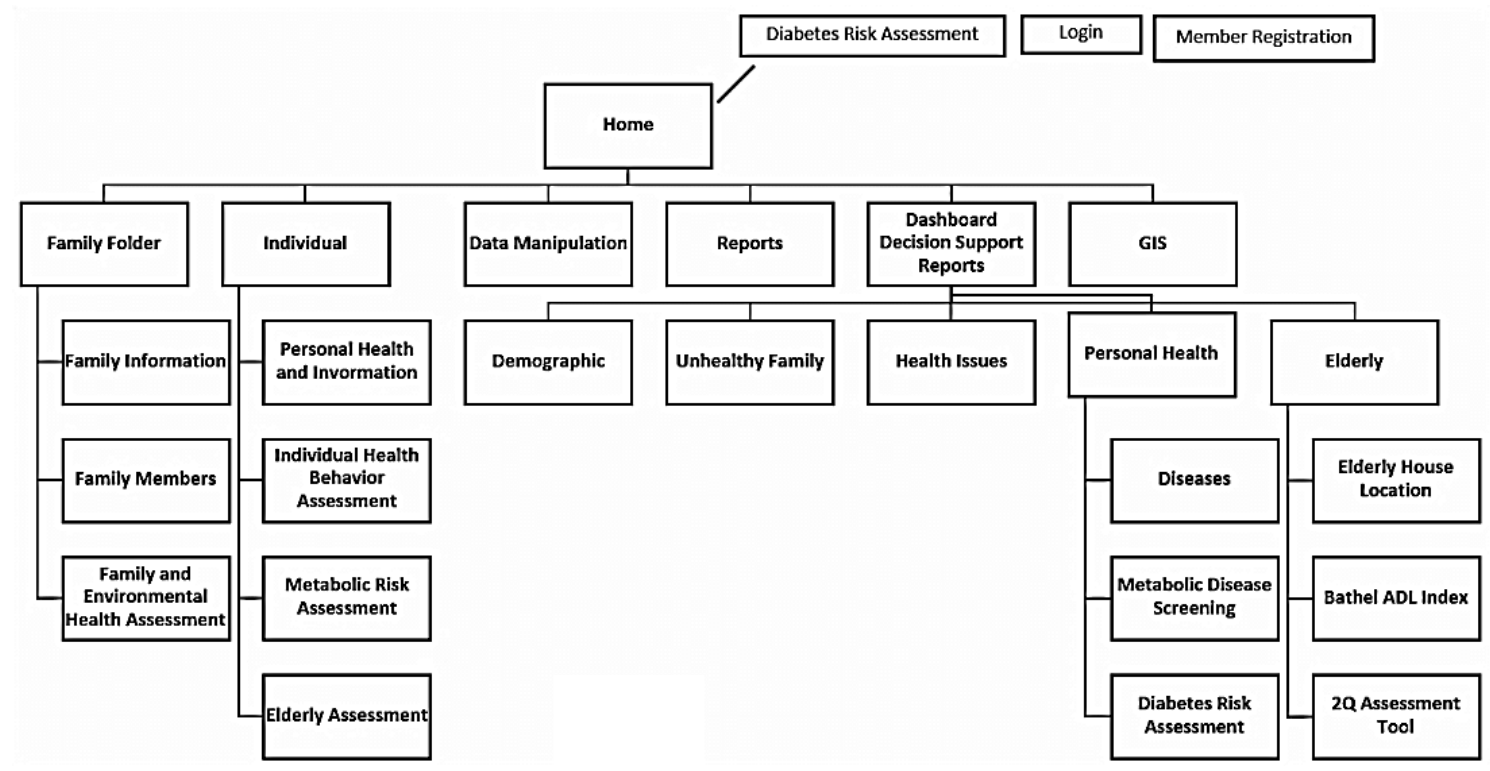

Figure 1 System overview and functional system summary; (a) DDSS overview and summary and (b) functional system summary. 


\section{Design and implementation of the DDSS}

The DDSS was developed using web-based technology, Business Intelligence (BI) concept, and open standard computing environments, i.e., MySQL [19], PHP [20], Highcharts JS [20], and Google Maps $[12,19]$. The DDSS uses a 3-tiered architecture (Figure 1a). The first tier is the user terminal, or presentation layer, where the user employs a browser to submit or input health data, the purpose of the report, and the source of the information, as well as to receive the transformed data, which the DDSS bases on criteria.

The second tier coordinates the applications, processes commands, makes logical decisions and evaluations, and performs calculations. This second tier moves and processes data between the first and third tiers. The form of visual representations is generated after the web server receives the performed data from the database server.

The third tier stores and retrieves data information from MySQL. Standard languages, such as SQL queries, are used for interaction with the second tier, using database specific protocols over TCP/IP. The generated information is then passed back to the second tier for processing, and then eventually sent back to the users on the first tier in the form of visual representations.

\section{Statistical analysis}

Internal consistency was tested using Cronbach's alpha to evaluate the degree of measurement error within each subscale. Acceptable values were greater than 0.700 . Content and constructive validity and reliability of the questionnaire were confirmed with Cronbach's alpha of 0.96 . We developed automatic calculation tools using statistical analysis concepts, such as percentages (\%) for nominal data and Chisquare tests for testing the differences between groups. A p-value of less than 0.05 was considered statistically significant. Ordinal data were described with mean values and standard deviations (SD) using SPSS.

\section{Usability evaluation}

User perception and effectiveness of the DDSS were evaluated using a questionnaire. The questionnaire was conducted according to ISO 9241-11 [21] combined with computer interaction design [22]. The questionnaire consisted of 32 questions under 7 main headings: effectiveness, efficiency, flexibility, learnability, memorability, errors/safety, and satisfaction. We selected 22 users from 3 organizations: (1) the CHLC at Thaioil (2) the hospital in Laem Chabang, and (3) the universities in Chonburi, covering all groups of users. The selected users were composed of (1) all of the executives and health officers of the CHLC, 2 executives and one health officer, (2) 3 staff from Laem Chabang hospital, and (3) one teacher and 15 students of the Faculty of Nursing, Burapha University. After the participants had used the DDSS for approximately one month, the questionnaire was given to users during a meeting. Participants were asked to fill out the questionnaire, indicating their level of agreement with a statement, using a 5-point Likert scale. The participants' anonymous responses were collected.

\section{Ethics statement}

This study was approved by the Ethical Clearance Committee on Human Rights Related to Researches Involving Human Subjects, Walailak University (016/2014).

\section{Results and discussion}

\section{System description}

The system was composed of 5 sections: family folder and family assessment tools, individual health assessment tools, decision support models and reports, Geographic Information System (GIS), and data manipulation. The system was designed to be used by 5 user groups: executives, practitioners, nursing interns and teachers, system administrators, and cooperation users. Users could access the system by logging into it. For security reasons, the login system required usernames and passwords obtained from the CHLC (Figure 1b). Since October 2010, a prototype DDSS has been available online at 
http://wjst.wu.ac.th

URL: http://thaioil.thaicommunityhealth.org/dashboardThaioil (Figure 2a). At present, the DDSS contains data from 14,328 individuals from 4,085 families in 10 communities.

(a)

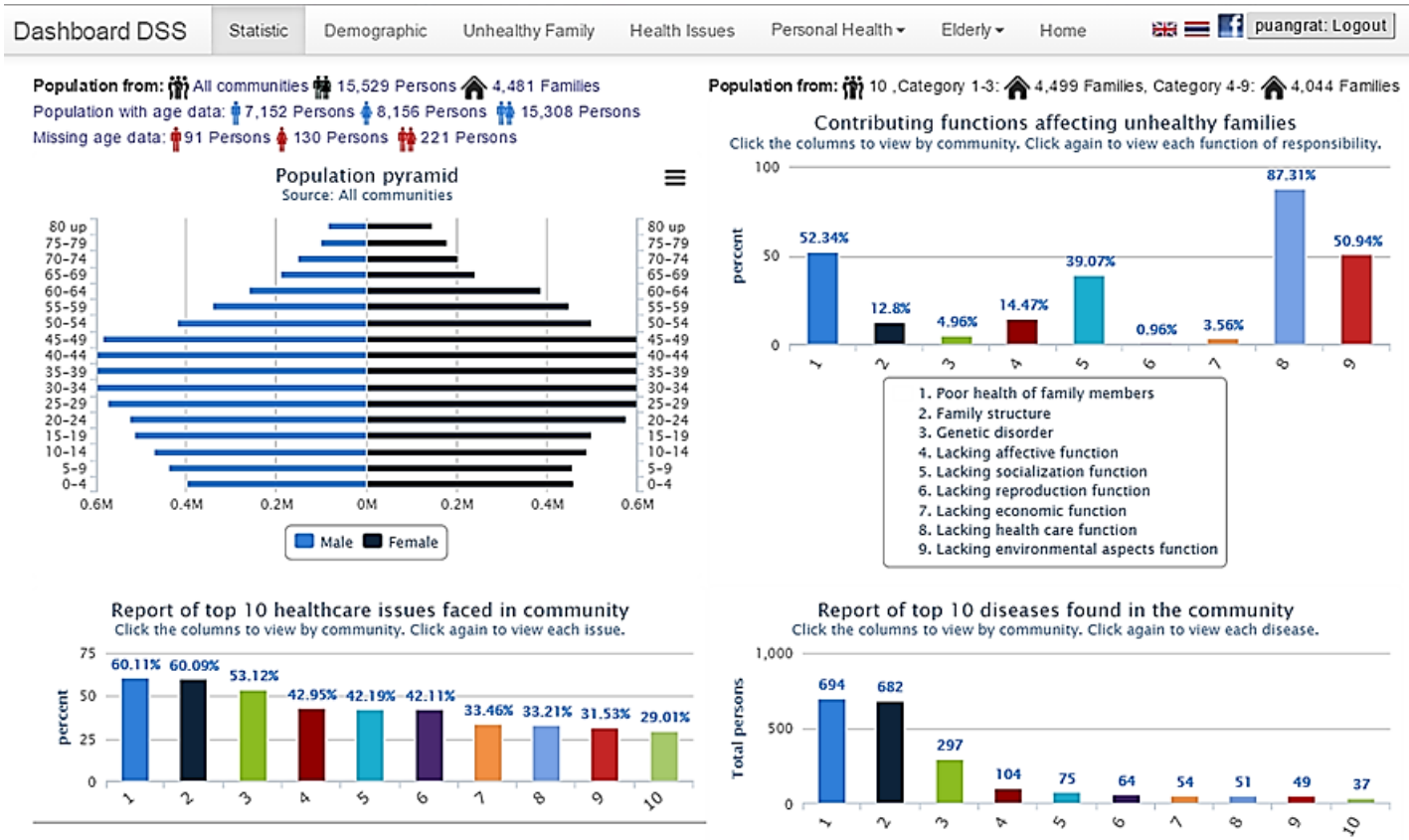

(b)

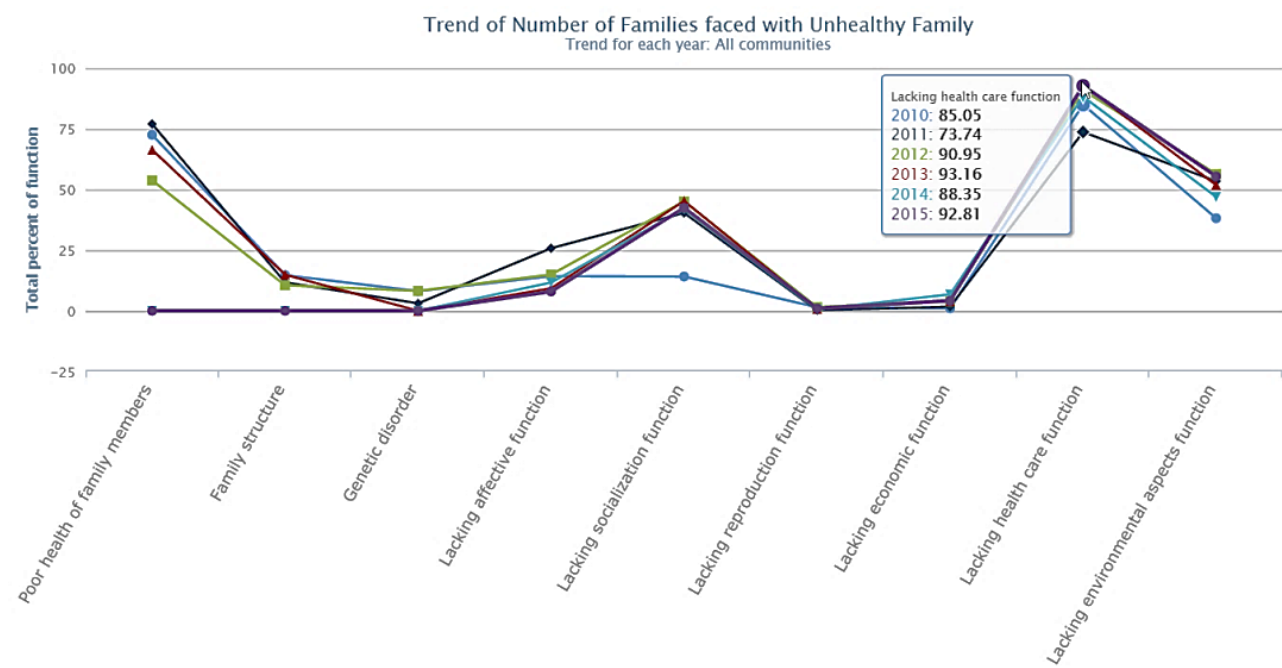

$-20 \overline{\overline{1}}$

Figure 2 DDSS home page and trend of families faced with dysfunctional family. (a) DDSS home page which shows population pyramid and bar chart of deficiency and (b) 6 years of data of families categorized as having problems of dysfunction. 
http://wjst.wu.ac.th

(a)

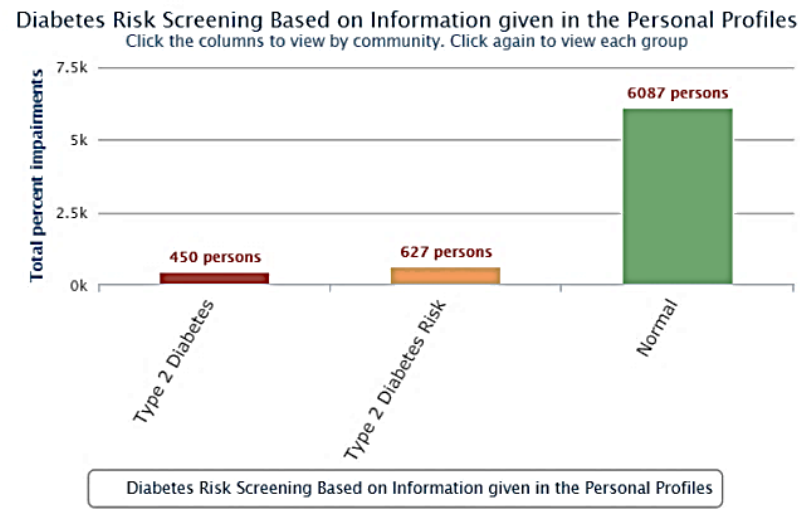

Percentage of Type 2 Diabetes

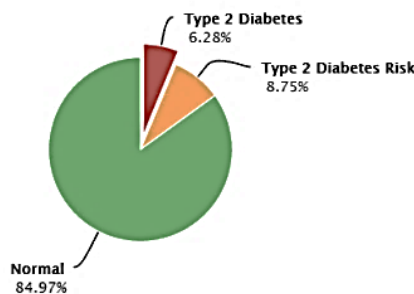

(b)

\begin{tabular}{|c|c|c|c|c|c|}
\hline \multirow[b]{2}{*}{ Gender } & \multicolumn{3}{|c|}{ Sell-dependent ability among the elderly: $O(\%)\left(E^{1}\right)\left(E^{2}\right)$} & \multirow[b]{2}{*}{ Sum (R) } & \multirow[b]{2}{*}{$\chi^{2}$} \\
\hline & No disability & Moderate disability & Severe disability & & \\
\hline Male & $294(97.35 \%)(100.67)(295.83)$ & $4(1.32 \%)(100.67)(3.99)$ & $4(1.32 \%)(100.67)(2.18)$ & 302 & $556.95 \cdots$ \\
\hline Female & $521(98.30 \%)(176.57)(519.17)$ & $7(1.32 \%)(176.67)(7.01)$ & $2(0.36 \%)(176.67)(3.82)$ & 530 & $1,006.76^{\cdots}$ \\
\hline Sum (c) & 815 & 11 & 6 & 832 & 2.41 \\
\hline
\end{tabular}

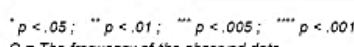

$O=$ The frequency of the obsened data

$E^{\prime}=$ The frequency of the expected values of gcodiness of fit (one-way) test

$E^{2}=$ The frequency of the expected values of independence (two-way) test

The contingency table represents the proportion of gender with ditferences in different levels of self-dependent ablilty (i.e. unable, need help and independent). The probabillties are less than conventional criteria for statistical significance $(P<.001)$ at the chi-squared distribution for 2 degree of freedoms. We conclude that there is evidence that elderly people who are of the same gender will have differences in self-dependent ability levels.

For the test of independence, the chi-squared statistic being less than the .05 critical point for statistical significance $\left(\chi_{2}^{2}=2.41, p>05\right)$. We conclude that there is evidence that elderly people who are different in gender will not have differences in self-dependent ability levels

(c)

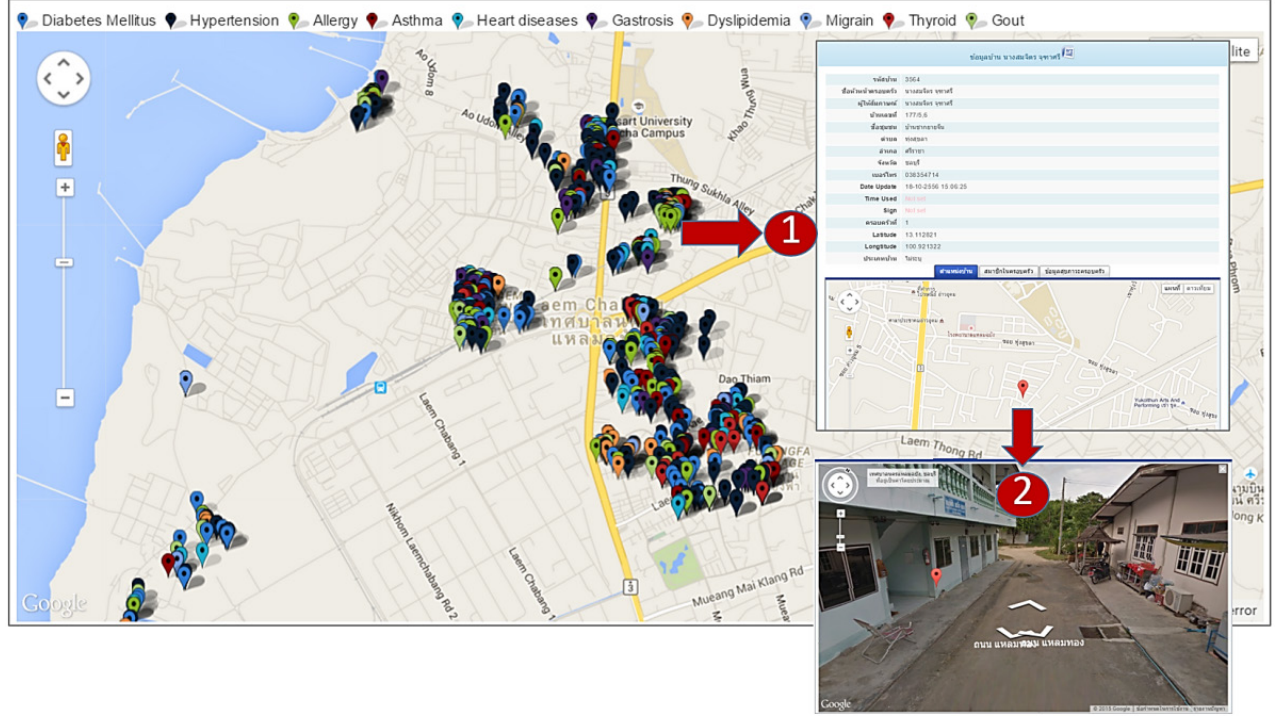

Figure 3 Reports for proactive health. (a) T2DM risk screening based on information given in the personal profiles, (b) the relationship between the ability to successfully complete certain activities without assistance with gender, using a Chi-Square test for independence, and (c) the prevalence of diseases distributed around communities. 


\section{Visualization and analytical tools}

The visualization tools provided demographical and statistical reports that summarized data determined to be essential for health promotion use. These tools were presented on dashboard in realtime, with aggregated data related to population and community health information including key performance indicators, to automatically calculate health status, and advanced statistical tools, such as the chi-square test.

The DDSS generated visual demographical reports in pyramid and stacked bar chart formats (Figure 2a). This tool was designed to present data in drill-down bar chart and data table formats to allow surveillance, and to obtain epidemiology data from top 10 statistical analyses of health issues and data on the prevalence of diseases faced in communities. In addition, the drill-down bar chart and data table format showed contributing functions affecting unhealthy families, in order to know the causes of problems within the family unit. Furthermore, the contributing functions affecting unhealthy families were represented in spider chart and line chart formats to see causes holistically and in terms of the trend of data over 6 years (Figure 2b). The data on metabolic disease screening were presented in 2 areas, (1) prevalence of metabolic disease and Type 2 Diabetes Mellitus (T2DM), and (2) categories of subjects at risk of T2DM. The results of this analysis are shown in Figure 3a in the form of bar and pie charts. In addition, we created a web-based T2DM risk assessment tool for self-assessment.

The automatic calculation of Chi-Square test was performed for classifications of self-dependent ability and gender; most elderly men and women were independent, and less than $2 \%$ of the elderly were classified in the severe disability group (Figure $\mathbf{3 b}$ ).

\section{DDSS through GIS visualization}

GIS data from the DDSS was used to visualize the location of houses in communities, the distributed locations of health conditions, such as houses which had encountered problems such as in each deficiency type, health issues, the prevalence of diseases, self-dependent ability in the elderly, and house location. Instead of presenting the raw data through tables or lists, we uploaded our presentation as a group of colorful balloons via Google maps (Figure 3c). Streets, places, and images surrounding the selected location could also be viewed in these maps.

\section{Evaluation of user satisfaction}

The overall average user satisfaction with the DDSS was 3.65. In terms of average value for each main heading of the questionnaire, comprising 7 main headings, user ratings were highest in satisfaction with (3.81) and effectiveness of (3.72) the system. The users also showed that they were concerned about the learnability of the system (2.75). Considering each main heading, in terms of the effectiveness of the system, users were satisfied that the DDSS delegated the authority groups for users (4.18). For efficiency and flexibility of the system, users were satisfied that the DDSS could provide results in a short time (3.59) and in many formats to meet their needs (3.59). When asked about learnability and memorability, they were satisfied that the DDSS assisted users to work easily, with no complicated processes (2.86), and that users could recognize the process to use to work with the DDSS (3.59). Users were moderately satisfied with the level of potential for errors in, and safety of, the system (3.18). In addition, they were satisfied with the design creativity of the user-program interaction (4.00). Finally, they reported that line graphs were the most useful way to present reports (4.00). Overall, the participating users found the DDSS useful in being a decision support system for health promotion (3.82).

Focusing on executives and health offices, 6 users from the CHLC and the hospital, we found that the overall average of user satisfaction with the DDSS was 4.30. The users suggested that they were most satisfied with the efficiency of the system (4.42). The users also showed that they were concerned about the level of potential for errors in, and safety of, the system (3.33). Focusing on another group of users, 16 users from the university, the evaluation indicated that the overall average of the user satisfaction with the DDSS was 3.41. The user ratings were highest in the overall satisfaction with the system (3.55). The users stated that they were most concerned about the learnability of the system (2.44). 


\section{Discussion}

The DDSS has data stored and visualized across several information systems, which are used to create health maps for improving health system performance and for planning proactive health care for the communities surrounding oil refinery. We have explored the design and usability of a visualization tool prototype based on ergonomics studies and computer interaction designs with visual display terminals. The findings of this research indicate that the DDSS is clearly usable for the purposes of a decision support system for health promotions. It also suggests that executives and health officers have increased awareness of health issue related data, and feel more in control of the situation.

The DDSS targets users with positions of executives and health officers, who need personal and community health information for the purposes of surveillance, obtaining epidemiology data, disease prevention, and disease control for communities served by a specific health facility [23,24]. The DDSS tool supports them to make better decisions for implementing programs in communities surrounding oil refinery as it can provide results in a short time and in many formats to meet their needs. In this study, we had one university professor and 15 nursing students from Burapha University testing and evaluating the DDSS tools with communities surrounding oil refinery. They found that by using the visualization system of the DDSS helps them analyze large data sets in a real time online fashion which in turn enhancing performance of decision-making in health promotion for communities around oil refinery. This webbased advanced tool generates effective programs in health promotion and disease prevention. Therefore, the DDSS, using graphical and numerical results, provides a better understanding of health situation in communities surrounding oil refinery [7,8]. This is in line with other researches suggesting that executives and health officers should use holistic, integrated and graphical displays for the quick interpretation of population and community health data when diagnosing situations of health, to proactively develop care plans $[1,9,10,12,25]$.

The integrated information visualization is a component to manage the amount of information without involving a complicated process, by presenting population and community health data in an easy to understand format $[8,9]$. Overall, satisfaction with the system was higher in executives and health officers when comparing with another group of users. This indicates that the DDSS is useful in the case of policy planning and managing programs. Learnability is a level of ease through which a user gains proficiency with the DDSS tools. Learnability of the DDSS tools can be evaluated through 3 methods (1) the amount of time to completion with the DDSS tools should be $10 \mathrm{~min}$ or less, (2) error rate and (3) the number of accurately completed the records. However, in this study, we did not investigated the learnability. We observed that users involved in data entry were concerned about the learnability of the system more than executives and health officers were, because they have many tasks dealing with the manipulation of data, especially the spatial data input. These results are consistent with other researches, which have shown that the presentation of information in different formats will have different effects on how information is evaluated $[25,26]$.

Distributed system problem types, developer usage modes, and the knowledge of users are distinctive strengths for the different approach of each visualization tool. The DDSS presents reports using static visualization techniques, such as pie, bar, and stack bar charts, spider maps, data tables, and GIS, to fit user requirements, ease of use and comprehension, and depth of report details. Similar static visualization techniques have been used in health care, using pie and bar charts and tables to conduct analyses in health care studies [27]. In additions, more animated tools, such as Gapminder, use bubble charts to visualize statistical time series, by converting numbers into animated and interactive graphics [28]. Despite users finding trend animation to be more fun and exciting, they only slightly preferred it to static depictions. Using animation to show trends in data clearly has value, especially concerning presentation. However, for analysis, static depictions of trends appear to be more effective.

This study has demonstrated how GIS can be used to assist health planners in identifying accessible sites for health examination and promotional programs that maximize the efficient utilization of resources $[12,19]$. The locating sites (Figure 3c) represent information on different areas of community member health status, such as presence of metabolic diseases, elderly person house locations, elderly person selfdependent abilities, and depression risks in the elderly; these were aided by the use of GIS. While others have reported the benefits of using the analytic operations of GIS to identify the geographic distribution 
http://wjst.wu.ac.th

of the population to be served, locate existing resources or services, and evaluate the spatial relationship between them, the GIS reports in our study used different colors of balloons to compare multiple values of factors. Health officers and community partnerships facilitated the selection of suitable sites for each report utilizing GIS [12,19].

There are several limitations associated with the development of this system. Firstly, the T2DM risk screening has analyzed and generated reports based on information given in the personal profiles without lab testing. This suggests that subjects at high risk of T2DM should be monitored and provided with proactive health activities, to save the costs of lab testing and to enhance their health. Secondly, the system needs internet access for GIS reports, due to Google maps being used for the geographic distribution of the population with attributed data. Thirdly, health officers have provided interventions for the communities, resulting from reports of this study. However, the consequences of such interventions are not included in this study. Finally, the number of subjects in each issue that could be investigated was limited by the frequency of assessment which interns have surveyed and inputted into the system.

\section{Conclusions}

The DDSS was developed and used to measure and create health maps for improving health system performance and for planning proactive health care for communities surrounding oil refinery. The results indicate that a highly interactive tool for integrated information visualization can potentially increase understanding of health status. In addition, it can integrate comprehensive health data to enhance decision-making and implement activities which can be created after complete understanding of the community health map has been reached. In the future, the result of deployment activity data and visit site data should be integrated into the system.

\section{Acknowledgements}

We thank John Barker and 2 anonymous referees for comments on previous versions of this manuscript, Urai Jaraprapal, and the Center of Excellence for Ecoinformatics, Walailak University, for knowledge support. We also thank nursing students of the Faculty of Nursing, Burapha University, and Boromarajonani College of Nursing, for their collaboration with this project. Financial support was obtained from the Thaioil Group Community Health and Learning Center, and the Southern College of Technology.

\section{References}

[1] JA Jacobs, E Jones, BA Gabella, B Spring and RC Brownson. Tools for implementing an evidencebased approach in public health practice. Prev. Chronic Dis. 2012; 9, 110324.

[2] World Health Organization. The Ottawa charter of health promotion, Available at: http://www.who.int/healthpromotion/conferences/previous/ottawa/en, accessed February 2015.

[3] GS Guldan. Obstacles to community health promotion. Soc. Sci. Med. 1996; 43, 689-95.

[4] C Merzel and JD Afflitti. Reconsidering community-based health promotion: Promise, performance, and potential. Am. J. Public Health 2003; 93, 557-74.

[5] The International Union for Health Promotion and Education (IUHPE). Available at: http://www.iuhpe.org/images/PROJECTS/ACCREDITATION/EHP_part1.pdf, accessed February 2015.

[6] AF Simpao, LM Ahumada, JA Galvez and MA Rehman. A review of analytics and clinical informatics in health care. J. Med. Syst. 2014; 38, 45.

[7] M Seah, MH Hsieh and P Weng. A case analysis of Savecom: The role of indigenous leadership in implementing a business intelligence system. Int. J. Inform. Manag. 2010; 30, 368-73.

[8] P Jinpon, M Jaroensutasinee and K Jaroensutasinee. Business intelligence and its applications in the public healthcare system. Walailak J. Sci. Tech. 2011; 8, 97-110.

[9] LM Sibley and HY Seow. Developing a dashboard to help measure and achieve the triple aim: A population based cohort study. BMC Health Serv. Res. 2014; 14, 363. 
http://wjst.wu.ac.th

[10] R Miniati, F Dori, G Cecconi, R Gusinu, F Niccolini and GB Gentili. HTA decision support system for sustainable business continuity management in hospitals: The case of surgical activity at University Hospital in Florence. Tech. Health Care 2013; 21, 49-61.

[11] PY Benhamou. Improving diabetes management with electronic health records and patients' health records. Diabetes Metab. 2011; 37, S53-S56.

[12] T Carlson, S York and J Primomo. The utilization of geographic information systems to create a site selection strategy to disseminate an older adult fall prevention program. Soc. Sci. J. 2011; 48, 15974.

[13] S Ahmed, SJ Bartlett, P Ernst, G Paré, M Kanter, R Perreault, R Grad, L Taylor and R Tamblyn. Effect of a web-based chronic disease management system on asthma control and health-related quality of life: Study protocol for a randomized controlled trial. Trials 2011; 12, 260.

[14] Thaioil Group. Communities Surrounding the Refinery. Available at: http://www.thaioilgroup.com/ home/content.aspx?id=144, accessed July 2015.

[15] P Wasi. Community Health System Development. Available at: http://www.prawase.com/images/ book/060800_PW_PB09.pdf, accessed July 2015.

[16] S Wibulpolprasert. Thailand Health Profile 2005-2007. Available at: http://www.moph.go.th/ops/ health_50, accessed July 2015.

[17] U Jaraeprapal. Pakpoon Subdistrict Administration Organisation with Community Well-Being development. Available at: http://resource.thaihealth.or.th/library/hot/12846, accessed July 2015.

[18] K Hachisuka, S Saeki, Y Tsutsui, H Chisaka, H Ogata, N Iwata and S Negayama. Gender-related differences in scores of the Barthel Index and Frenchay activities index in randomly sampled elderly persons living at home in Japan. J. Clin. Epideminol. 1999; 52, 1089-94.

[19] B Zuberbuhler, P Galloway, A Reddy, M Saldana and R Gale. A web-based information system for management and analysis of patient data after refractive eye surgery. Comput. Meth. Prog. Bio. 2007; 88, 210-6.

[20] MS Hutton, S Azevedo, R Beeler, R Bettenhausen, E Bond, A Casey, J Liebman, A Marsh, T Pannell and A Warrick. Experiment archive, analysis, and visualization at the National Ignition Facility. Fusion Eng. Des. 2012; 87, 2087-91.

[21] ISO 9241-11. Ergonomic Requirements for Office Work with Visual Display Terminals, Part 11: Guidance on Usability. Available at: https://www.iso.org/obp/ui/\#iso:std:iso:9241:-11:ed-1:v1:en, accessed July 2015.

[22] J Preece, Y Rogers and H Sharp. In: Proceeding of the Interaction Design: Beyond HumanComputer Interaction, New York, 2002, p. 169-82.

[23] N Amek, P Vounatsou, B Obonyo, M Hamel, F Odhiambo, L Slutsker and K Laserson. Using health and demographic surveillance system (HDSS) data to analyze geographical distribution of socio-economic status: An experience from KEMRI/CDC HDSS. Acta Trop. 2015; 144, 24-30.

[24] L Botetzagias, AF Dima and C Malesios. Extending the theory of planned behavior in the context of recycling: The role of moral norms and of demographic predictors. Resour. Conservat. Recycl. 2015; 95, 58-67.

[25] J Forsman, N Anani, A Eghdam, M Falkenhav and S Koch. Integrated information visualization to support decision making for use of antibiotics in intensive care: Design and usability evaluation. Inform. Health Soc. Care 2013; 38, 330-53.

[26] ST Hawley, B Zikmund-Fisher, P Ubel, A Jancovic, T Lucas and A Fagerlin. The impact of the format of graphical presentation on health-related knowledge and treatment choices. Patient Educ. Couns. 2007; 73, 448-55.

[27] MA Thomas, PR Narayan and C Christian. Mitigating gaps in reproductive health reporting in outlier communities of Kerala, India: A mobile phone-based health information system. Health Pol. Tech. 2012; 1, 69-76.

[28] Gapminder. About Gapminder. Available at: http://www.gapminder.org/about-gapminder, accessed June 2015. 